\title{
Evidence that urocortin is absent from neurons of the Edinger-Westphal nucleus in pigeons
}

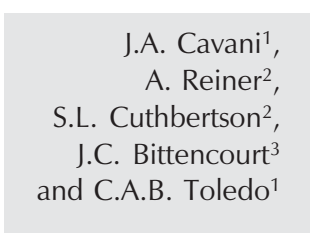

\author{
${ }^{1}$ Laboratório de Neurociências, Universidade Cidade de São Paulo, \\ São Paulo, SP, Brasil \\ ${ }^{2}$ Department of Anatomy and Neurobiology, The University of Tennessee, \\ Memphis, TN, USA \\ ${ }^{3}$ Departamento de Anatomia, Universidade de São Paulo, São Paulo, SP, Brasil
}

\section{Correspondence \\ C.A.B. Toledo \\ Laboratório de Neurociências \\ Universidade Cidade de São Paulo \\ Rua Cesário Galeno, 448 \\ 03071-000 São Paulo, SP \\ Brasil \\ Fax: +55-11-6190-1300 \\ E-mail: ctoledo@unicid.br}

Presented at the XVIII Annual Meeting of the Federação de Sociedades de Biologia Experimental, Curitiba, PR, Brazil, August 27-30, 2003.

Research supported by FAPESP (Nos. 02/01919-3 to J.A. Cavani, and 00/04536-2 to C.A.B. Toledo) and $\mathrm{NIH}$ (EY-05298 to A. Reiner).

....................

Received June 24, 2003

Accepted September 9, 2003 .....................

\begin{abstract}
The Edinger-Westphal nucleus (EWN) is a central preganglionic parasympathetic cell group that gives rise to cholinergic input to the ciliary ganglion, thereby regulating several neurovegetative ocular functions. Recently, the supposed presence of the neuropeptide urocortin (UCN) has been reported in EWN neurons in rodent brain. The purpose of the present study was to examine the distribution of UCN in avian brain and to investigate by immunohistochemical analysis the possible use of this substance as an EWN marker in a nonmammalian class of vertebrates. Brain tissue of pigeons was incubated with a specific antibody against UCN and the results showed labeling of many small neurons, forming a double wing in the dorsal mesodiencephalic transition area. Their size and shape, however, differed from those of EWN neurons, and they were preferentially located rostral to the EWN. Double-label experiments employing an antibody against the enzyme choline acetyltransferase (ChAT) showed that UCN is not localized to the cholinergic cells of the EWN and confirmed the rostral distribution of UCN never overlapping the ChAT+ EWN cells. Taken together, these results suggest that, at least in pigeons, the $\mathrm{UCN}+$ population does not belong to the traditionally defined EWN.
\end{abstract}

Key words

- Urocortin

- Avian brain

- Visual system

- Oculomotor complex

- Comparative neuroanatomy
The Edinger-Westphal nucleus (EWN) of birds is the autonomic part of the oculomotor complex and the source of preganglionic inputs to the ciliary ganglion (1-4). In avian species, the EWN can be divided into two portions, lateral and medial, which are distinct in cell size and shape and in their physiological roles. In pigeons, the lateral portion (EWL) consists of bigger and more spherical cells that project to ciliary neurons of the ciliary ganglion, giving rise to the ciliary nerve innervating the ciliary body and the iris sphincter muscle (1-4). The ciliary neurons mediate pupilloconstriction and accommodation. The medial portion of the EWN (EWM), in turn, innervates choroidal neurons of the ciliary ganglion, which project to the choroid and regulate its blood flow $(4,5)$.

According to Gamlin and Reiner (3), the major afferent pathways to the avian EWN are: a) the predominantly contralateral path- 
way from the suprachiasmatic nucleus in the hypothalamus, which terminates in the EWM, b) the pathway from the medial mesencephalic reticular formation to both EWM and EWL neurons, c) the pathway beginning in the rostral lateral mesencephalic reticular formation projecting to the EWL, and d) the pathway which proceeds from a pretectal nucleus, the area pretectalis, and projects contralaterally to the caudolateralmost part of the EWL.

In mammals, the functional subdivisions of the EWN are not as clear and its limits are still controversial (6). Recently, the substance urocortin (UCN) was reported to be expressed in EWN neurons of rodents, suggesting it to be a potential marker for this nucleus (7-9). However, UCN was also present in cells of some other regions of the brain, such as the supraoptic and paraventricular nuclei of the hypothalamus, anterior pituitary, substantia nigra, and cerebellum $(7,10,11)$. In mammals, UCN neurons in the EWN area were also reported to project to brain districts not related to ocular adjustments, such as the lateral septal nucleus and spinal cord (8). Since the avian EWN is well characterized and well defined cytoarchitectonically, the purpose of the present study was to ascertain if UCN is present in EWN neurons of birds. By knowing both the precise anatomical and physiological characteristics of the EWN, the determination of the UCN labeling pattern in birds might provide important information concerning the UCN system in mammals. We carried out a series of immunohistochemical experiments using a specific antibody against UCN (8) to map its localization in the avian brain.

Twelve pigeons (Columba livia) were deeply anesthetized by intramuscular injections of xylazine $(0.1 \mathrm{mg} / 100 \mathrm{~g})$ and ketamine $(0.2 \mathrm{mg} / 100 \mathrm{~g})$ and perfused with saline followed by $4 \%$ paraformaldehyde in $0.1 \mathrm{M}$ sodium phosphate buffer ( $\mathrm{pH}$ 7.4). Brains were removed and cryoprotected by 24-h immersion in a solution containing $30 \%$ sucrose in phosphate buffer. Using a sliding microtome, $35-\mu \mathrm{m}$ thick frozen coronal sections were obtained and collected into separate compartments. Tissue was rinsed in phosphate buffer and incubated overnight with the polyclonal antibody against $\mathrm{UCN}$ at room temperature (rabbit anti-UCN, generously provided by Joan Vaughan and Wylie Vale from the Peptide Biology Laboratory, The Salk Institute for Biological Studies, La Jolla, CA, USA (8)). To identify the cholinergic EWN neurons, we also used a polyclonal antiserum against choline acetyltransferase (ChAT) (goat anti-ChAT from Chemicon International Inc., Temecula, CA, USA). After washing in phosphate buffer, the tissue was incubated with the secondary biotinylated antibody (donkey anti-rabbit for UCN experiments and donkey anti-goat for ChAT, both from Jackson ImmunoResearch Labs., West Grove, PA, USA) for $1 \mathrm{~h}$, followed by a new wash in phosphate buffer. The tissue was then incubated with the avidin-biotincomplex (ABC-Elite kit, Vector Laboratories, Burlingame, CA, USA) for one additional hour. Labeling was visualized with a standard diaminobenzidine protocol (4), and sections were mounted on slides, dehydrated and coverslipped. For histological mapping, alternate sections that had not been immunostained were processed by the Giemsa technique. Results were observed and analyzed with a Nikon E-800 microscope, using the atlas of the pigeon brain of Karten and Hodos (12) to identify and define structures. Data were photographed on Kodak-Ektachrome film or the images were captured with a CCD camera (Magnafire, Optronics, Goleta, CA, USA). All procedures were conducted in accordance with the Brazilian College of Animal Experimentation policies on the ethical use of animals in research.

After Giemsa staining, EWN neurons formed a compact mass of cells immediately dorsal to the oculomotor nucleus $(\mathrm{OM})$. They were identified by their typical ovoid shape (Figure 1D). The immunohistochemical stud- 
ies (Figure 1A,B) demonstrated that, in general, cells that expressed UCN in the pigeon brain were fusiform and medium-sized (ranging from 8 to $27 \mu \mathrm{m}$ at their major axis; mean, $16.2 \mu \mathrm{m}$ ), contrasting with the archetypal spherical shape of the EWN cells, as can be seen in Figure 1D. The major UCNcontaining cell group was shaped like a wedge lying rostral to the OM, situated mostly medial to the EWN-OM complex (Figures 1 and 2). This main $\mathrm{UCN}$-positive $(\mathrm{UCN}+)$ population consisted of paired cell columns, positioned in the diencephalic-mesencephalic transition zone, along the ventral aspect of the substantia grisea centralis (just below the cerebral aqueduct), and ending almost at the nucleus ruber level (Figure 1). At that level, as can be perceived in Figure 1, the dorsal portion of the UCN+ nucleus appears to abut the medial aspect of the nucleus interstitialis (nucleus of Cajal), with a location corresponding to that of the nucleus of Darkschewitsch (ND) of birds (12). The ND

Figure 1. The distribution of urocortin- (UCN) containing neurons in pigeon brain and in the nucleus of Edinger-Westphal (EWN). Digital images of coronal sections of the mesodiencephalic transition area in the pigeon brain. With a specific antibody against UCN many UCN+ neurons may be seen forming two medial columns (indicated by arrows) located at the dorsal portion of the substantia grisea centralis ( $A$ and B). The main group of the UCN-labeled neurons forms a wing-like structure just below the central aqueductus, at the caudal hypothalamus. The image in $B$ presents a detail of the UCN+ neuron position, medially running over the nucleus interstitialis (IS). The next serial section stained with Giemsa (C) permitted us to identify that the pigeon brain area where $\mathrm{UCN}+$ neurons were found corresponds to the nucleus of Darkschewitsch (ND). The image in D represents a caudal level of the pigeon brain stained with Giemsa where the neurons of the oculomotor complex formed by the dorsal and ventral portions of the oculomotor nucleus (OMd and OMv, respectively) and the EWN are observed. At the level of the images in $A, B$, and $C$, no OM or EWN neurons can be seen. Magnification bars: $500 \mu \mathrm{m}$ ( $A$ and $D)$ and $200 \mu \mathrm{m}$ ( $B$ and $C$ ). Other abbreviations: $F R M=$ formatio reticularis medialis mesencephali; $\mathrm{RU}=$ nucleus ruber.
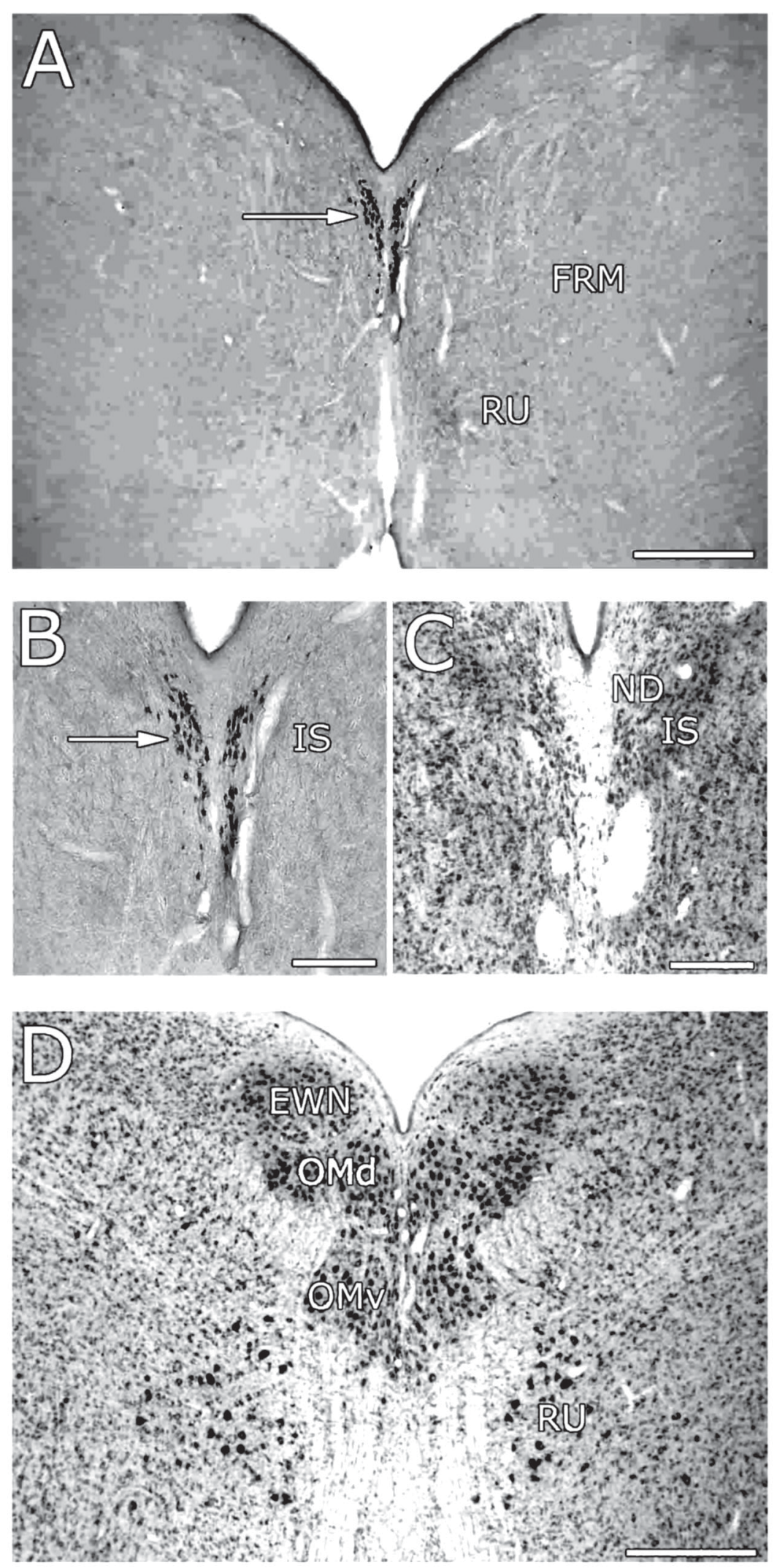
of pigeons is formed by a tight cluster of medium to small cells (mean, $14.1 \mu \mathrm{m}$ ), mostly fusiform in shape, abutting the medial side of the nucleus interstitialis, matching the positioning described for UCN+ neurons. Information regarding the ND dimension in birds is not readily available and to specifically determine its limits, cell typology and chemical characteristics, it would be necessary to perform further cytoneurochemical analyses. Nevertheless, according to our current data, the UCN+ neurons seem to constitute at least part of the ND in pigeons.

For rats, Bittencourt et al. (8) reported that the main cluster of UCN+ neurons is located medial to the ND and between the two cell columns of the OM. The same report indicates that the most rostral UCN+

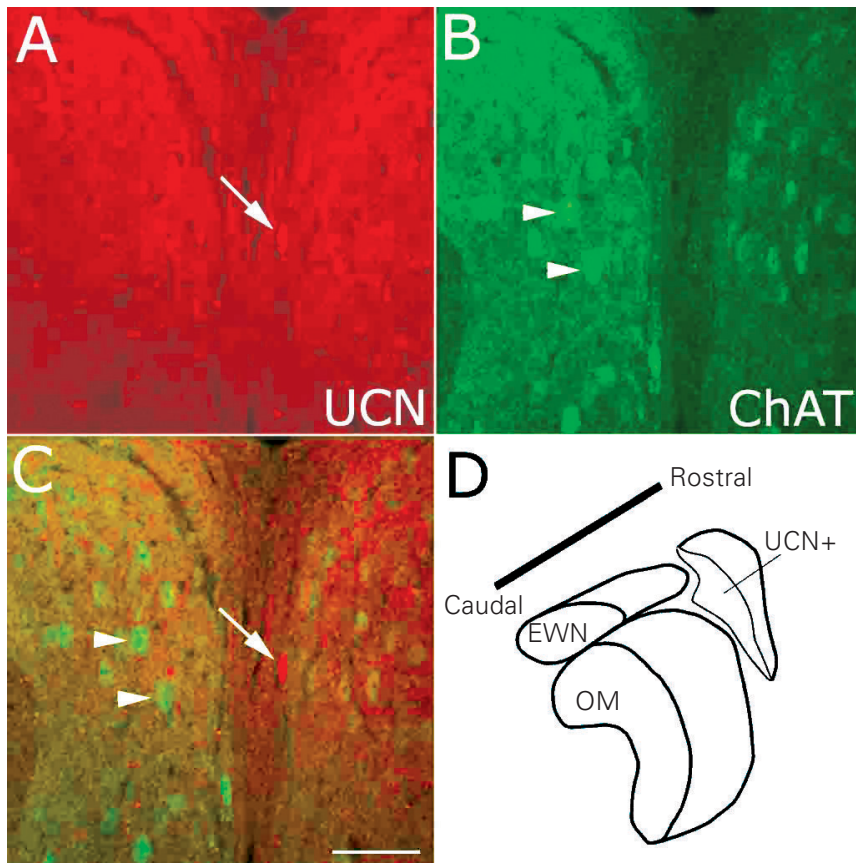

Figure 2. Urocortin-containing neurons are not cholinergic. Digital image of fluorescence label for urocortin ( $A, U C N)$ and the enzyme choline acetyltransferase (B, ChAT). The brain level shown in $A, B$, and $C$ corresponds to the caudalmost portion of the UCN+ main group, where the $\mathrm{UCN}+$ cells are rare. ChAT neurons (arrowheads) are distinct and bigger than UCN neurons (arrows). The merged image (C) shows that the markers clearly do not localize in the same cell. At bottom right (D), a unilateral drawing of the location of the main UCN group as regarding the oculomotor nucleus (OM) and Edinger-Westphal nucleus (EWN) position is presented. Magnification bar: $50 \mu \mathrm{m}$. neurons in rats are situated at hypothalamic levels well rostral to the OM. These $\mathrm{UCN}+$ neurons are reportedly located medial to the mammillotegmental tract and to the interstitial nucleus of Cajal. These findings are consistent with the present results, although some UCN+ cells tended to spread laterally in the vicinity of the EWN at the caudal end of the UCN field (Figure 2D). At that point, the caudalmost extent of the ND, a few $\mathrm{UCN}+$ neurons were localized medial to the EWN and OM, in a position reminiscent of the UCN+ cells in relation to the OM complex in mammals, but none of them lie within the EWN in birds. Even the few UCN+ cells medially located at the level of the EWN, tended to be smaller than those constituting the EWN-OM complex.

In birds, the EWN neurons (as well as the OM motor neurons) are virtually all cholinergic (4). To confirm that the UCN is not found in EWN neurons a double-label immunofluorescence experiment was performed with a primary antibody against UCN (8) and a polyclonal antiserum against ChAT (Chemicon). In such cases, we employed secondary antibodies marked with different fluorophores: fluorescein isothiocyanate and tetramethylrhodamine isothiocyanate, observed using an epifluorescence microscope (Nikon E-800, Tokyo, Japan). The results of this experiment confirmed that cells expressing UCN are different in size and shape from those of the EWN and demonstrated that the $\mathrm{UCN}$ is not located in cholinergic cells of the EWN or OM (Figure 2A,B,C). These data allowed us to conclude that, in birds, the EWN (defined as the autonomic part of the $\mathrm{OM}$ that controls specific parasympathetic functions of the eye via the ciliary ganglion) does not contain UCN.

Thus, by anatomical criteria, UCN+ neurons in pigeons do not seem to be part of the EWN. This finding helps reconcile the seeming paradox created by reports that the EWN, a cell group known to be involved in parasympathetic control of ocular functions, con- 
tains UCN+ neurons. UCN is a corticotrophin-releasing hormone agonist, triggering responses through a class of corticotrophinreleasing hormone receptors $(8,13-17)$. $\mathrm{UCN}+$ neurons appear to be part of the brain circuitry involved in sympathetic nervous system-mediated behavioral responses to stress (14,17-19). The distribution of the $\mathrm{UCN}+$ neurons as a column arising at hypothalamic levels and projecting to the spinal cord (7) is consistent with these interpretations. The present results for birds suggest that while the UCN+ neurons may be near the EWN, they are not part of it. Thus, sympathetic systemic functions and parasympathetic ocular functions do not appear to be mediated by the same cell group, EWN, at least in birds.

In birds, neurons with a distribution resembling that of the UCN+ neurons described here have been shown to project to the spinal cord (20). Thus, the UCN+ neurons near the avian EWN appear to have the same projection as those in mammals, suggesting that they are indeed the same population of neurons, with presumably a similar role in stressrelated responses. Nonetheless, studies directly examining the possible spinal projection of the UCN cells are desirable, particularly of their precise target within the cord. In any event, the evidence that the UCN+ neurons do project to the spinal cord in birds reinforces the view that the mammalian midbrain may contain separate populations of neurons as well - UCN+ neurons projecting to the cord involved in systemic sympathetic functions and cholinergic preganglionic neurons projecting to the ciliary ganglion involved in parasympathetic ocular functions.

Since UCN-containing neurons are not cholinergic and the proposed functions of UCN do not correspond to the ocular reflexes controlled by the EWN, current evidence suggests that the $\mathrm{UCN}+$ population forms a distinct group. Our anatomical data also indicate that the bulk of the UCN+ neurons in pigeons lie rostral to the $\mathrm{OM}$ complex. Thus, the UCN+ cells are not truly part of the avian EWN as classically defined, i.e., the source of preganglionic input to the ciliary ganglion, and this seems likely to be true for mammals as well.

\section{Acknowledgments}

The authors wish to thank Claudio Osiris de Oliveira for valuable help during the experimental procedures, Marcia Tsuruta for technical support, and Drs. Joan Vaughan and Wylie Vale, from the The Salk Institute, CA, USA, for kindly providing the antibody against urocortin.

\section{References}

1. Reiner A, Karten HJ, Gamlin PDR \& Erichsen JT (1983). Parasympathetic ocular control: functional subdivisions and circuitry of the avian nucleus of Edinger-Westphal. Trends in Neurosciences, 6: 451-472

2. Gamlin PDR, Reiner A, Erichsen JT, Karten HJ \& Cohen DH (1984). The neural substrate of the pupillary light reflex in the pigeon (Columba livia). Journal of Comparative Neurology, 226: 523-543.

3. Gamlin PDR \& Reiner A (1991). The Edinger-Westphal nucleus: sources of input influencing accommodation, pupilloconstriction, and choroidal blood flow. Journal of Comparative Neurology, 306: 425-438

4. Reiner A, Erichsen JT, Cabot JB, Evinger C, Fitzgerald MEC \& Karten HJ (1991). Neurotransmitter organization of the nucleus of Edinger-Westphal and its projection to the avian ciliary ganglion. Visual Neuroscience, 6: 451-472.
5. Fitzgerald MEC, Gamlin PDR, Zagvzdin Y \& Reiner A (1996). Central neural circuits for the light-mediated reflexive control of choroidal blood flow in the pigeon eye: a laser Doppler study. Visual Neuroscience, 13: 655-669.

6. May PJ, Porter JD \& Gamlin PDR (1992). Interconnections between the primate cerebellum and midbrain near-response regions. Journal of Comparative Neurology, 315: 98-116.

7. Yamamoto H, Maeda T, Fujimura M \& Fujimiya M (1998). Urocortinlike immunoreactivity in the substantia nigra, ventral tegmental area and Edinger-Westphal nucleus of rat. Neuroscience Letters, 243 $21-24$

8. Bittencourt JC, Vaughan J, Arias C, Rissman RA, Vale WW \& Sawchenko PE (1999). Urocortin expression in rat brain: evidence against a pervasive relationship of urocortin-containing projections with targets bearing type 2 CRF receptors. Journal of Comparative 
Neurology, 415: 285-312.

9. Kozicz T (2003). Neurons colocalizing urocortin and cocaine and amphetamine-regulated transcript immunoreactivities are induced by acute lipopolysaccharide stress in the Edinger-Westphal nucleus in the rat. Neuroscience, 116: 315-320.

10. Oki Y, Iwabuchi M, Masuzawa M, Watanabe F, Ozawa M, Ino K, Tominaga T \& Yoshimi T (1998). Distribution and concentration of urocortin, and effect of adrenalectomy on its content in rat hypothalamus. Life Sciences, 62: 807-812.

11. Shi M, Yan X, Ryan DH \& Harris RB (2000). Identification of urocortin mRNA antisense transcripts in rat tissue. Brain Research Bulletin, 53: 317-324.

12. Karten HJ \& Hodos W (1967). A Stereotaxic Atlas of the Brain of the Pigeon (Columba livia). Johns Hopkins University Press, Baltimore, MD, USA

13. Asaba K, Makino S \& Hashimoto K (1998). Effect of urocortin on $\mathrm{ACTH}$ secretion from rat anterior pituitary in vitro and in vivo: comparison with corticotropin-releasing hormone. Brain Research, 806: 95-103.

14. Koob GF \& Heinrichs SC (1999). A role for corticotropin releasing factor and urocortin in behavioral responses to stressors. Brain
Research, 848: 141-152.

15. Weninger SC, Peters LL \& Majzoub JA (2000). Urocortin expression in the Edinger-Westphal nucleus is up-regulated by stress and corticotropin-releasing hormone deficiency. Endocrinology, 141: 256-263.

16. Skelton KH, Owens MJ \& Nemeroff CB (2000). The neurobiology of urocortin. Regulatory Peptides, 93: 85-92.

17. Smagin GN, Heinrichs SC \& Dunn AJ (2001). The role of CRH in behavioral responses to stress. Peptides, 22: 713-724.

18. Weninger SC, Dunn AJ, Muglia LJ, Dikkes P, Miczek KA, Swiergiel AH, Berridge CW \& Majzoub JA (1999). Stress-induced behaviors require the corticotropin-releasing hormone $(\mathrm{CRH})$ receptor, but not $\mathrm{CRH}$. Proceedings of the National Academy of Sciences, USA, 96: 8283-8288.

19. De Fanti BA \& Martinez JA (2002). Central urocortin activation of sympathetic-regulated energy metabolism in Wistar rats. Brain Research, 930: 37-41.

20. Cabot JB, Reiner A \& Bogan N (1982). Avian bulbospinal pathways: anterograde and retrograde studies of cells of origin, funicular trajectories and laminar terminations. Progress in Brain Research, 57: 79-108. 